\title{
Heat stability of proteins and its exploitation for purification of heat-stable proteins
}

\author{
Muhammad Ahmad ${ }^{1}$, Aisha Tahir ${ }^{2}$, and Rana Salman Anjum ${ }^{*}$ \\ ${ }^{1}$ School of Life Sciences, Forman Christian College (A Chartered University), Lahore, Pakistan \\ ${ }^{2}$ Department of Biochemistry, University of Health Sciences, Lahore, Pakistan \\ *Corresponding author: salmananjum@fccollege.edu.pk
}

\begin{abstract}
:
Proteins possess complex three-dimensional structures, and these structures are stable only within specific ranges of temperature which mostly correspond to the temperature ranges of the host organisms. However, few exceptional proteins, called heat-stable proteins, are stable at temperatures that are substantially higher than those tolerated by the host organisms themselves. Most of the heat-stable proteins possess heat stability to perform their functions at high temperatures, but some of them are intrinsically heat-stable due to their structure. Heat-stable proteins are usually divided into three or four groups depending upon the intricacies of their structures and thermal behaviors. Their peculiar property, i.e. heat-stability, makes them very valuable in applications such as polymerase chain reaction, industrial processes requiring high temperature, and protein engineering. Heat-stability also makes it feasible to purify such proteins, from the rest of the heat-labile proteins, using a simple heat-treatment method. Moreover, heat treatment can be used as a combined cell-lysis and protein purification step which, as compared to conventional methods, can result in a higher yield of heat-stable proteins. Furthermore, some special heat-stable proteins, i.e. intrinsically disordered proteins (which include the proteins involved in important neurodegenerative diseases), need heat-treatment step, in some cases, as the only way for their successful purification and study. Hence, this paper provides a first-ever comprehensive review of all major aspects of heat-stable proteins, i.e., their structure, evolution, classification, significance, and heat-treatment mediated purification.
\end{abstract}

Keywords: Heat-stable proteins, Heat treatment, Hyperthermophilic proteins, Heat stability, Protein purification 


\section{Denaturation of proteins:}

Proteins are complex macromolecules that play structural and functional roles in all types of living organisms. The function of a protein is dependent upon its structure, so a change in structure will usually lead to a change in function or even loss of function. As depicted in Fig. 1A, change in the structure of a protein is called denaturation if it involves alterations in hydrogen bonding and other non-covalent interactions of protein which can result in a state of the protein that is less-ordered (its $\alpha$-helices changed into extended $\beta$-sheets) or unfolded (Bischof \& He, 2006; Chaplin, 2001; David, 2005). However, denaturation does not encompass the breakage of covalent bonds that interconnect atoms of the polypeptide. Besides, denaturation should not be confused with the phenomena of aggregation or coagulation. These two phenomena are different from denaturation but usually happen following denaturation. Aggregation is defined as the general association of proteins with each other to form large complexes and coagulation is the random joining of denatured proteins. Denatured proteins usually undergo aggregation both in vivo and in vitro (Bischof \& He, 2006; David, 2005). Studies have shown that protein aggregates in cells contain both native and denatured proteins (Bischof \& He, 2006). Moreover, native proteins can maintain their activities in aggregate forms and can be disaggregated, into single active native proteins, by cell while recovering from shock (Wallace et al., 2015).

\section{Mechanism of denaturation of proteins:}

Denaturation of a protein is not always complete and irreversible. Proteins can be denatured completely or partially. In addition, their denaturation can be irreversible as well as reversible (Bischof \& He, 2006). For instance, denaturation caused by low temperature is usually reversible denaturation. The structure of a protein also decides whether it will be denatured reversibly or irreversibly. Groups of (heat-stable) proteins that are denatured reversibly and those that are denatured irreversibly, along with the method to figure out the nature of denaturation (Kim et al., 2000), will be discussed later in this review. Bischof \& He (2006) and David (2005), while discussing the thermodynamics of protein denaturation, state that denaturation of a protein does not happen until the activation energy of denaturation is provided. Overcoming the activation energy results in the conversion of the native state of a protein into its denatured state which usually has a higher energy level than the native state. This denatured state of the protein can, then, undergo 
highly exothermic processes of aggregation or coagulation which always results in a state of lower energy level than the native state of the protein (Fig. 1B).

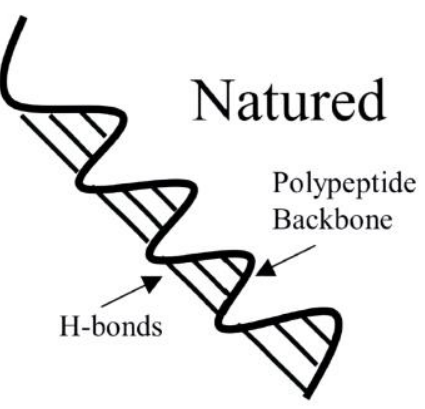

A.


Fig. 1. Protein denaturation and its thermodynamics. (A) Hydrogen bonds of an $\alpha$-helix are broken down during denaturation. (B) Natured/native state is converted, upon the availability of activation energy, into a denatured state having higher energy that can, then, release energy to change into a lower energy aggregated/coagulated state. (Both figures are cartoon representations and not drawn to scale). Adapted from "Thermal stability of proteins," by J.C. Bischof and X. He, 2006, Annals of the New York Academy of Sciences, 1066(1), p.15, Copyright 2005 by New York Academy of Sciences. Adapted with permission.

\section{Heat-induced denaturation of proteins:}

Every protein can maintain its native structure only within its specified temperature range. Outside that temperature range, denaturation of protein, i.e., unfolding transition, starts. Onset temperature is the lowest temperature at which denaturation/unfolding of a protein occurs, e.g., $40-45^{\circ} \mathrm{C}$ is the general onset temperature for mammalian cells. The width of the unfolding transition is usually $10-12{ }^{\circ} \mathrm{C}$, and the midpoint temperature of this transition is called the transition temperature or 
melting temperature $\left(\mathrm{T}_{\mathrm{m}}\right) . \mathrm{T}_{\mathrm{m}}$ is the midpoint of a protein's thermal unfolding curve (Fig. 2). At $\mathrm{T}_{\mathrm{m}}$ of a protein, half of that protein has been unfolded, so $\mathrm{T}_{\mathrm{m}}$ of a protein is a measure of its thermal stability (Argos, 1979; Bischof \& He, 2006; David, 2005).

\section{The temperature dependence of the heat capacity and denaturation}

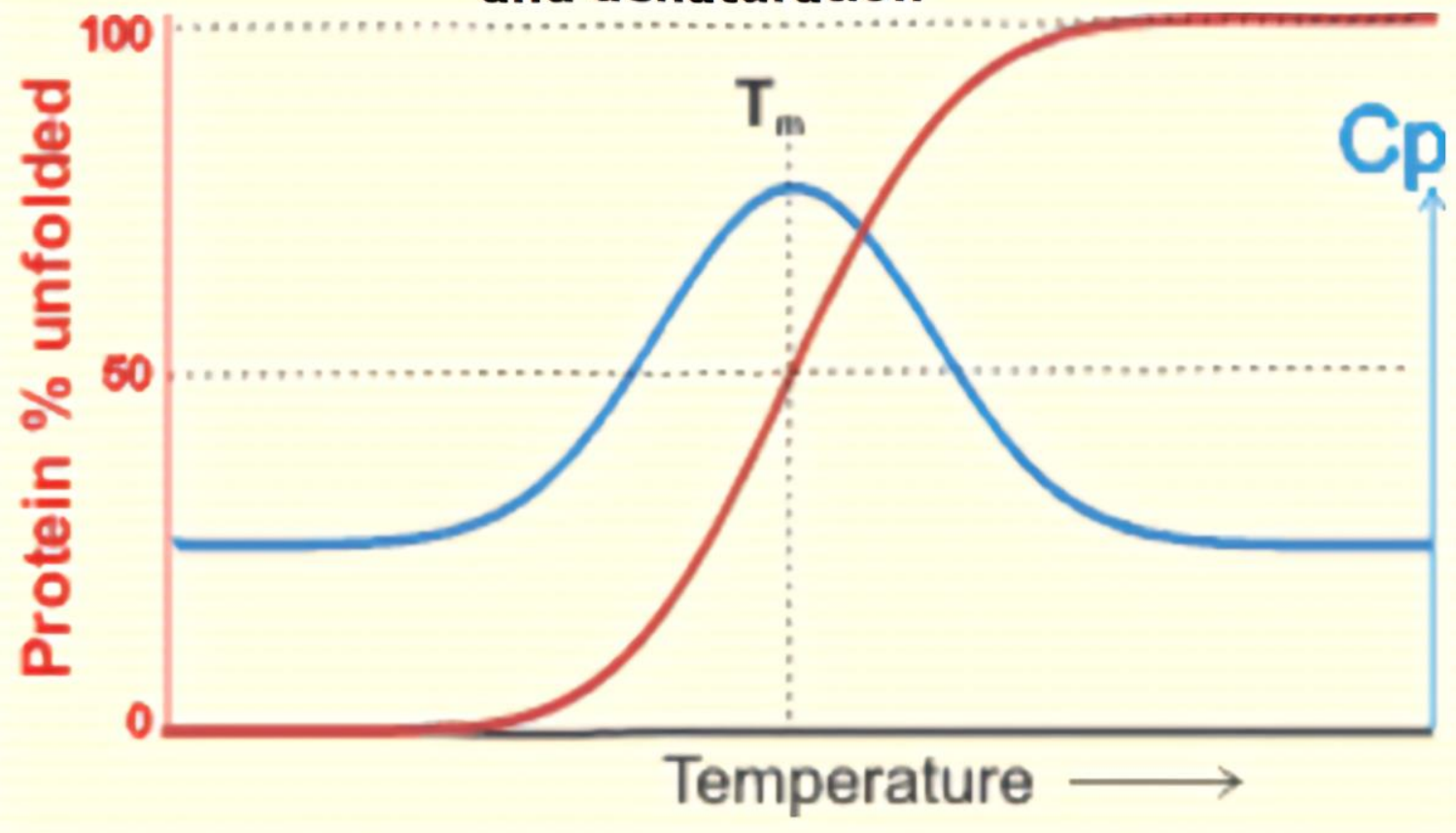

Fig. 2. The thermal unfolding curve of protein. $T_{m}$ is the temperature at which half of a protein is folded, while its other half is unfolded. Adapted from Protein Folding and Denaturation, In London South Bank University, by M. Chaplin, 2001, from http://www1.1sbu.ac.uk/water/protein_denatured.html. Copyright 2001 by Martin Chaplin. Adapted with permission. 
Most of the proteins are heat labile, so they undergo denaturation and aggregation upon heat treatment. In contrast, some proteins, called heat-stable proteins/heat-resistant proteins, remain in their soluble state upon heating (Kim et al., 2000). At this point, it is also useful to differentiate a bit between heat shock proteins and heat-stable proteins. Heat shock proteins are the proteins whose expression is induced or increased upon exposure of the host organism to high temperatures (Schumann et al., 2016). On the other hand, heat-stable proteins, which are the focus of our review and discussed below in detail, are the proteins that remain stable at high temperatures (Kim et al., 2000). The evolutionary reasons for the heat-stability of heat-stable proteins, classification of heatstable proteins, and applications of heat-stable proteins are described below.

\section{The reasons behind the evolution of heat-stable proteins:}

Generally, the temperature range in which proteins belonging to an organism are stable and functional is linked to the temperature of the environment in which that organism lives. For this reason, psychrophilic organisms possess proteins that are stable only at low temperatures and are readily denatured even at moderate temperatures. In contrast, proteins from hyperthermophilic organisms can remain stable at very high temperatures, i.e., $100{ }^{\circ} \mathrm{C}$ and above. This allows hyperthermophilic organisms to survive in habitats with very high temperatures because their proteins, including enzymes, can perform their respective functions at or above the boiling point of water. It may seem counterintuitive that although mesophilic organisms live at moderate temperatures, they are well-known to possess heat-stable proteins. These proteins can tolerate temperature that can be very higher than can be tolerated by their host organisms. For example, some proteins from Escherichia coli can remain stable even at $100{ }^{\circ} \mathrm{C}$, while these bacteria themselves cannot grow at $50^{\circ} \mathrm{C}$ or above. A possible explanation for excessive heat tolerance of such heat-stable proteins, from mesophilic organisms, is that these proteins may have heat stability just due to nature of their structure (as in case of intrinsically disordered proteins, etc.), or this heat stability may allow these proteins to perform their specialized functions, e.g., acting as heat shock proteins, etc. (Kim et al., 2000; Kwon et al., 2008; Paz et al., 2010).

\section{Classification of heat-stable proteins:}


Kwon et al. (2008) gave heat-treatment, from $20{ }^{\circ} \mathrm{C}$ to $85^{\circ} \mathrm{C}$ and back to $20^{\circ} \mathrm{C}$, to proteins from E. coli and proposed a general classification of the obtained heat-stable proteins into three classes (based on the different ways in which these classes of proteins can remain soluble when they are given heat treatment). The first class of heat-stable proteins has a very rigid structure that is not unfolded by heat treatment, so these proteins remain in their native and soluble state upon heating. Proteins from thermophilic/hyperthermophilic organisms probably belong to this class of proteins. The second class of heat-stable proteins unfolds upon heating, but their unfolded state is soluble in water. The third class of heat-stable proteins is unfolded by heating but can refold to their native state when cooled down (Fig. 3).

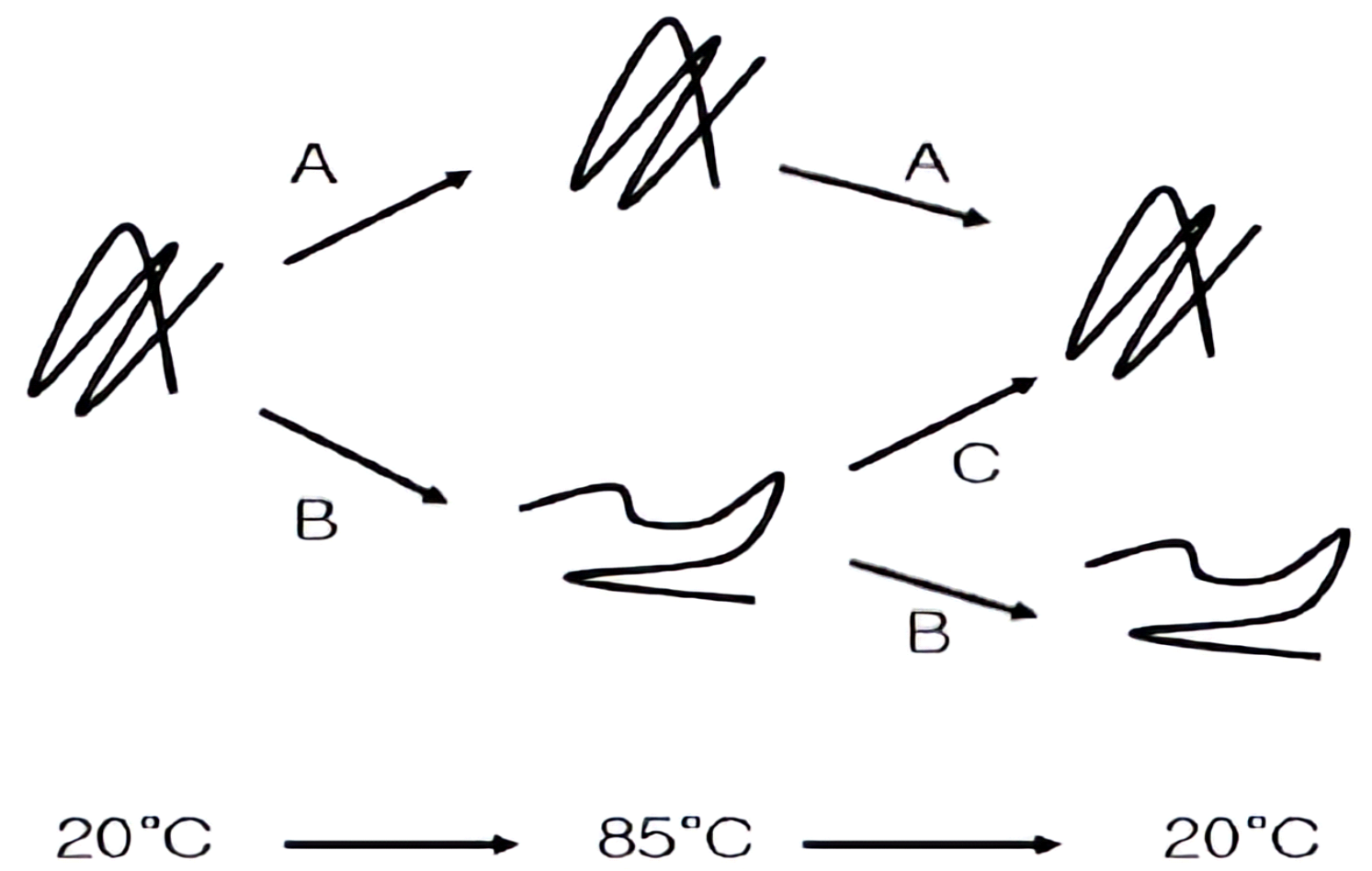

Fig. 3. A general classification of heat-stable proteins. Heat-stable proteins are classified into three classes depending upon the paths adopted by them, to remain soluble, upon heat-treatment. The first class retains their native soluble state (path A), the second class is converted into an unfolded soluble state (path B), and the third class unfolds but, upon cooling, refolds to their native soluble state (path C). Adapted from "Proteomic analysis of heat-stable proteins in Escherichia coli," by S. Kwon, Y. Jung, and D. Lim, 2008, BMB reports, 41(2), p.110, Copyright 2008 by Korean Society for Biochemistry and Molecular Biology. Adapted with permission.

A more profound classification of heat-stable proteins was done by Kim et al. (2000) on the basis of signals from Circular Dichroism (CD) Spectroscopy when heat-stable proteins were heated from 
$20^{\circ} \mathrm{C}$ to $100{ }^{\circ} \mathrm{C}$ and, then, cooled back from $100{ }^{\circ} \mathrm{C}$ to $20^{\circ} \mathrm{C}$. $\mathrm{CD}$ signals were taken as a measure of heat-induced secondary structural changes in proteins, and this method allowed them to classify heat-stable proteins into 4 groups. $1^{\text {st }}$ group of heat-stable proteins consisted of intrinsically disordered proteins that are devoid of any secondary structure (and will be discussed later in this review). CD signals of this group had a linear relationship with change in temperature. CD spectra of $2^{\text {nd }}$ group of proteins showed that they were mainly $\alpha$-helical polypeptides. They unfolded upon heat treatment, and heat-induced unfolding of these proteins was irreversible. CD spectra of the $3^{\text {rd }}$ group of heat-stable proteins indicated that they were $\beta$-stranded proteins. They also unfolded upon heat treatment, but the heat-induced unfolding of these proteins was reversible. $4^{\text {th }}$ group of heat-stable proteins consisted of a hyperthermophilic protein (protein from a hyperthermophilic organism), i.e., glutamate racemase, from a hyperthermophilic bacteria, in this case. Its CD spectrum showed that it had a finely-ordered secondary structure. Moreover, no change in the CD spectrum, indicating no change in the structure, of this protein was observed until the melting temperature, but this protein precipitated and gave no $\mathrm{CD}$ signal above $100{ }^{\circ} \mathrm{C}$. The behavior of this protein showed that hyperthermophilic proteins had thermal behavior similar to that of heatlabile proteins because both types of proteins denatured and precipitated at temperatures above their $\mathrm{T}_{\mathrm{m}}$ values. The only difference between these two types of proteins was that hyperthermophilic proteins had much higher $\mathrm{T}_{\mathrm{m}}$ values than heat-labile proteins, and this difference allowed hyperthermophilic proteins to maintain their structure at temperatures a lot higher than that tolerated by heat-labile proteins. No such protein, belonging to the $4^{\text {th }}$ group of heat-stable proteins, has been found in humans (Kim et al., 2000). The behavior of heat-labile proteins and different classes of heat-stable proteins, upon heat treatment, is manifested in the figure below (Fig. 4). 


\title{
Thermal behavior of proteins
}

\section{Heat-labile proteins}

\author{
Native $\longrightarrow$ Unfolded $\longrightarrow$ Precipitation

\section{Heat-resistant proteins}
1) Group $I$
3) Group III
Unstructured $\longleftrightarrow$ Unfolded
Native $\longleftrightarrow$ Unfolded
2) Group II
4) Group IV

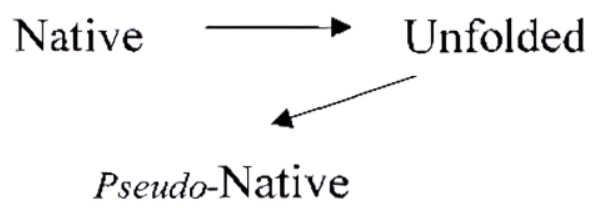
Native $\longleftrightarrow$ Native

Figure. 4. Comparison among thermal behaviors of heat-labile and different groups of heatstable proteins. Upon heat treatment, heat-labile proteins irreversibly unfold and precipitate, and thus are converted into their insoluble forms. On the other hand, heat-stable proteins, without losing their stability, either remain in native/pseudo-native states or convert into their reversible unfolded states. Adapted from "Thermal Behavior of Proteins: Heat-Resistant Proteins and Their HeatInduced Secondary Structural Changes," by T.D. Kim, H.J. Ryu, H. I. Cho, C.H. Yang, and J. Kim, 2000, Biochemistry, 39(48), p.14845, Copyright 2000 by American Chemical Society. Adapted with permission.

\section{Significance of heat-stable proteins:}

The exceptional heat stability of heat-stable proteins makes them very useful in many research and industrial settings. As compared to other proteins, which require maintenance at low temperature during handling and storage, heat-stable proteins don't require such care by researchers. Heatstable proteins are also used in research protocols, like the polymerase chain reaction, that requires proteins to remain active at high temperatures. Moreover, heat-stable enzymes are valuable in catalyzing industrial processes that have to be carried out at high temperatures. These industrial processes include chemical synthesis and pulp bleaching; processing of food, starch, leather, and textile; production of pharmaceuticals, ethanol, detergents, and cosmetics, etc. Furthermore, knowledge of structural features of heat-stable proteins is used by protein engineers to produce heat-stable variants of functionally important heat-labile proteins (Bae et al., 2008; Unsworth et 
al., 2007). Besides, heat-stable bacteriocins are important in food preservation (Park et al., 2002). Finally, intrinsically disordered proteins, which are a special class of heat-stable proteins, play very important roles in cells due to their unique structural features. Therefore, misfolding of these proteins can result in many human disorders particularly in neurodegenerative disorders including Alzheimer's disease, Huntington disease, Parkinson's disease, dementia with Lewy body, Down's syndrome, amyotrophic lateral sclerosis, and many more (Raychaudhuri et al., 2009; Uversky, 2015).

\section{Benefits of exploiting heat stability of heat-stable proteins for their purification:}

Many researchers took advantage of the heat stability of heat-stable proteins for simplifying their purification. It was done by heating total protein extract, from different organisms, at an appropriate temperature, and for a sufficient amount of time, to denature most of the proteins other than the protein of interest. These denatured proteins could include all heat-labile proteins and some heat-stable proteins that are more easily denatured by the heat than the protein of interest. Denatured proteins would aggregate and pelletize upon centrifugation, while the protein of interest, being a heat-stable protein, would remain soluble in the supernatant upon centrifugation. In this way, a heat-stable protein can be easily purified, to homogeneity in some cases, by just heat treatment and centrifugation (Olichon et al., 2007). Besides, $\mathrm{pH}$ and other components of a buffer, such as the concentration of reducing agents, sugars, and polyols, are very important factors for heat treatment, and they can be manipulated to increase denaturation of contaminating proteins and/or preserve the concentration of (heat-stable) protein of interest (Back et al., 1979; Law \& Leaver, 2000). Benefits, along with few drawbacks, of using heat treatment step for purification of heat-stable proteins are described below:

\section{No or less chromatography (or other purification) steps required for purification:}

Techniques that are generally used for protein purification, such as different chromatography techniques, are very costly and time-consuming (Sahoo et al., 2009). On the other hand, heat treatment is a very simple step that can be used, as an alternative method, for effective purification 
of heat-stable proteins. In some cases, just heat treatment was enough to purify heat-stable proteins to homogeneity, and no other purification technique was required. For instance, using heat treatment as the only step for purification, Lapidot et al. (1996) achieved >98\% homogeneity of heat-stable xylanase, from Bacillus stearothermophilus, that was overexpressed in Escherichia coli. Similarly, Oka et al. (1989) used only heat treatment to get $>95 \%$ homogeneity of heat-stable leucine dehydrogenase, also from B. stearothermophilus, that was also overexpressed in E. coli. However, in most cases, heat treatment proved to be very effective for partial purification of heatstable proteins, but one (Moure et al., 2012) or more (Cukier-Kahn et al., 1972; Josse, 1966; Khandelwal \& Zinman, 1978; Rosen et al., 1971; Sahoo et al., 2009; Sieg et al., 1996) chromatography (or other purification) steps were required, following heat treatment step, for complete purification of heat-stable proteins. Hence, heat treatment could be used as a short, easy, cheap, and efficient step for initial purification of heat-stable proteins which, if required, could be further purified by other methods of protein purification.

Nonetheless, heat treatment has a drawback that it can affect the structure and activity of the purified protein (Kalthoff, 2003; Moure et al., 2012). Thus, before choosing heat treatment as a standard method for purification of a heat-stable protein, the protein should be purified with and without heat treatment step, and properties of both types should be compared to determine whether heat treatment affected any property of the protein. In this regard, the work of Moure et al. (2012) provided an exemplary experimental design. They compared structure (by NMR analysis) and function (by protein-protein interaction study) of GlnB, purified with and without heat treatment step, and proved that heat treatment, along with only one chromatography step, could be used as a quick protocol to purify GlnB without introducing any alteration in conformation, chemical composition, or activity of the protein.

\section{Greater yield of heat-stable protein and no need of separate lysis:}

These two benefits of heat treatment were elucidated by the work of Kalthoff (2003) who used heat treatment for purification of unstructured (and, thus, heat-stable) domains of two proteins, i.e., epsin 1 and AP180, that were recombinantly expressed in E. coli. He used heat treatment for bacterial lysis and, as a control, he lysed another bacterial culture using Triton X-100 and 
sonication. Results of his study showed that $72 \%$ of epsin 1's domain and 70\% of AP 180's domain were recovered by lysis caused by heat treatment. In the case of control, protein recovery was just $29 \%$ and $37 \%$ for domains of epsin 1 and AP 180, respectively. It showed that a major improvement in protein recovery occurred by using heat treatment, instead of sonication, for lysis. Moreover, heat treatment very effectively purified these proteins' domains from most of the other proteins. Thus, according to his study, heat treatment is not only an effective purification step, but it can also be used simultaneously as a lysis step which results in increased recovery, in comparison to lysis by sonication, of heat-stable unstructured proteins. However, he cautioned that high temperature can cause chemical modifications in amino acids of proteins, e.g., by Maillard reaction (Kalthoff, 2003; Paz et al., 2010), so lysis buffer should be chosen accordingly and, as an initial check, properties of proteins obtained by heat treatment method should be compared with same proteins purified by other methods (Kalthoff, 2003).

\section{Protection against proteases (especially in case of intrinsically disordered proteins):}

Heat treatment can also protect heat-stable proteins from proteases' action by causing denaturation of these proteases. It is especially helpful in the case of intrinsically disordered proteins, which are also heat-stable proteins, for their easy and effective purification. These proteins, due to their disordered and extended/open structure, are highly heat-stable and also highly prone to proteolysis. The problem of high susceptibility to proteases can be usually solved by carrying out purification at low temperatures and in the presence of an appropriate mixture of protease inhibitors. But, in some cases, both of these strategies fail to prevent the degradation of intrinsically disordered proteins. The heat treatment step can be very helpful in these cases. As a result of heat, proteases, being globular proteins, will be denatured while intrinsically disordered proteins, being heat-stable proteins, will survive. Heating will also denature heat-labile proteins which will result in easy purification of intrinsically disordered proteins. So, one of the best and easiest ways to prevent proteolysis of intrinsically disordered proteins, during purification, is to purify intrinsically disordered proteins by heat treatment method (Kalthoff, 2003; Paz et al., 2010).

\section{Conclusion:}


Heat-stable proteins allow thermophilic/hyperthermophilic organisms to live and reproduce at very high temperatures, while these proteins, by acting as heat shock proteins, protect mesophilic organisms from high-temperature stress. Based on their thermal behaviors, heat-stable proteins can be categorized into four groups. These proteins have immense significance in medical, industrial, and research settings, and they need to be purified for their studies and applications. Due to their heat-stability, they can be simply and effectively purified by heat-treatment method which has tremendous benefits compared to other purification strategies. The knowledge and significance of heat-stable proteins, presented in this review, is intended to accelerate future research in this field.

\section{References:}

Argos P, Rossmann MG, Grau UM, Zuber H, Frank G, Tratschin JD (1979). Thermal stability and protein structure. Biochemistry; 18(25): 5698-5703.

Bae E, Bannen RM, Phillips GN (2008). Bioinformatic method for protein thermal stabilization by structural entropy optimization. Proceedings of the National Academy of Sciences; 105(28): 9594-9597.

Bischof JC \& He X (2006). Thermal stability of proteins. Annals of the New York Academy of Sciences; 1066(1): 12-33.

Chaplin, M (2001). Protein Folding and Denaturation. London South Bank University. http://www1.1sbu.ac.uk/water/protein_denatured.html

Cukier-Kahn R, Jacquet M, Gros F (1972). Two heat-resistant, low molecular weight proteins from Escherichia coli that stimulate DNA-directed RNA synthesis. Proceedings of the National Academy of Sciences; 69(12): 3643-3647.

David W (2005). Proteins: structure and function. Chichester: John Wiley \& Sons Ltd. 52-58, 396-398, 401-403.

Kalthoff C (2003). A novel strategy for the purification of recombinantly expressed unstructured protein domains. Journal of Chromatography B; 786(1-2): 247-254.

Khandelwal RL, Zinman SM (1978). Purification and properties of a heat-stable protein inhibitor of phosphoprotein phosphatase from rabbit liver. Journal of Biological Chemistry; 253(2): 560-565. 
Kim TD, Ryu HJ, Cho HI, Yang CH, Kim J (2000). Thermal behavior of proteins: Heatresistant proteins and their heat-induced secondary structural changes. Biochemistry; 39(48): $14839-14846$.

Kwon S, Jung Y, Lim D (2008). Proteomic analysis of heat-stable proteins in Escherichia coli. Journal of Biochemistry and Molecular Biology; 41(2): 108-111.

Lapidot A, Mechaly A, Shoham Y (1996). Overexpression and single-step purification of a thermostable xylanase from Bacillus stearothermophilus T-6. Journal of biotechnology; 51(3): 259-264.

Law AJ, Leaver J (2000). Effect of $\mathrm{pH}$ on the thermal denaturation of whey proteins in milk. Journal of Agricultural and Food Chemistry; 48(3): 672-679.

Moure VR, Razzera G, Araújo LM, Oliveira MA, Gerhardt EC, Müller-Santos M, ..., Huergo LF (2012). Heat stability of Proteobacterial PII protein facilitate purification using a single chromatography step. Protein expression and purification; 81(1): 83-88.

Oka M, Yang YS, Nagata S, Esaki N, Tanaka H, Soda K (1989). Overproduction of thermostable leucine dehydrogenase of Bacillus stearothermophilus and its one-step purification from recombinant cells of Escherichia coli. Biotechnology and applied biochemistry; 11(3): 307-311.

Olichon A, Schweizer D, Muyldermans S, de Marco A (2007). Heating as a rapid purification method for recovering correctly-folded thermotolerant $\mathrm{VH}$ and $\mathrm{VHH}$ domains. BMC biotechnology; 7(1): 7 .

Park C, Zhou S, Gilmore J, Marqusee S (2007). Energetics-based protein profiling on a proteomic scale: identification of proteins resistant to proteolysis. Journal of molecular biology; 368(5): 1426-1437.

Park SY, Yang YJ, Kim YB, Hong JH, Lee C (2002). Characterization of subtilein, a bacteriocin from Bacillus subtilis CAU131 (KCCM 10257). Journal of microbiology and biotechnology; 12(2): 228-234.

Paz A, Zeev-Ben-Mordehai T, Sussman JL, Silman I (2010). Purification of intrinsically disordered proteins. Instrumental Analysis of Intrinsically Disordered Proteins: Assessing Structure and Conformation; 695-704. 
Raychaudhuri S, Dey S, Bhattacharyya NP, Mukhopadhyay D (2009). The role of intrinsically unstructured proteins in neurodegenerative diseases. PloS one; 4(5): e5566.

Sahoo D, Andersson J, Mattiasson B (2009). Immobilized metal affinity chromatography in open-loop simulated moving bed technology. Purification of a heat stable histidine tagged $\beta$ glucosidase. Journal of chromatography B; 877(16-17): 1651-1656.

Schumann W (2016). Regulation of bacterial heat shock stimulons. Cell Stress and Chaperones; 21(6): 959-968.

Sieg F, Schroder W, Schmitt JM, Hincha DK (1996). Purification and characterization of a cryoprotective protein (cryoprotectin) from the leaves of cold-acclimated cabbage. Plant physiology; 111(1): 215-221.

Unsworth LD, van der Oost J, Koutsopoulos S (2007). Hyperthermophilic enzymes - stability, activity and implementation strategies for high temperature applications. The FEBS journal; 274(16): 4044-4056.

Uversky VN (2015). Intrinsically disordered proteins and their (disordered) proteomes in neurodegenerative disorders. Frontiers in aging neuroscience; 7: 18.

Wallace EW, Kear-Scott JL, Pilipenko EV, Schwartz MH, Laskowski PR, Rojek AE, ..., Airoldi EM (2015). Reversible, specific, active aggregates of endogenous proteins assemble upon heat stress. Cell; 162(6): 1286-1298. 